\title{
Delay in solar energetic particle onsets at high heliographic latitudes
}

\author{
S. Dalla ${ }^{1}$, A. Balogh ${ }^{1}$, S. Krucker ${ }^{2}$, A. Posner ${ }^{3}$, R. Müller-Mellin ${ }^{3}$, J. D. Anglin ${ }^{4}$, M. Y. Hofer ${ }^{5}$, R. G. Marsden ${ }^{5}$, T. R. \\ Sanderson $^{5}$, B. Heber ${ }^{6}$, M. Zhang ${ }^{7}$, and R. B. McKibben ${ }^{8}$ \\ ${ }^{1}$ Blackett Laboratory, Imperial College, London, UK \\ ${ }^{2}$ Space Sciences Laboratory, University of California, Berkeley, USA \\ ${ }^{3}$ University of Kiel, Germany \\ ${ }^{4}$ National Research Council of Canada, Ottawa, Canada \\ ${ }^{5}$ Research and Scientific Support Dept. of ESA, ESTEC, The Netherlands \\ ${ }^{6}$ University of Osnabrück, Germany \\ ${ }^{7}$ Florida Institute of Technology, USA \\ ${ }^{8}$ University of New Hampshire, USA
}

Received: 12 November 2002 - Revised: 27 February 2003 - Accepted: 3 March 2003

\begin{abstract}
Ulysses observations have shown that solar energetic particles (SEPs) can easily reach high heliographic latitudes. To obtain information on the release and propagation of SEPs prior to their arrival at Ulysses, we analyse the onsets of nine large high-latitude particle events. We measure the onset times in several energy channels, and plot them versus inverse particle speed. This allows us to derive an experimental path length and time of release from the solar atmosphere. We repeat the procedure for near-Earth observations by Wind and SOHO. We find that the derived path lengths at Ulysses are 1.06 to 2.45 times the length of a Parker spiral magnetic field line connecting the spacecraft to the Sun. The time of particle release from the Sun is between 100 and $350 \mathrm{~min}$ later than the release time derived from in-ecliptic measurements. We find no evidence of correlation between the delay in release and the inverse of the speed of the CME associated with the event, or the inverse of the speed of the corresponding interplanetary shock. The main parameter determining the magnitude of the delay appears to be the difference in latitude between the flare and the footpoint of the spacecraft.
\end{abstract}

Key words. Interplanetary physics (energetic particles) Solar physics, astrophysics and astronomy (energetic particles, flares and mass ejections)

\section{Introduction}

The passages of the Ulysses spacecraft over the poles of the Sun in 2000 and 2001, during solar maximum conditions, provided measurements of solar energetic particles (SEPs) at high heliographic latitudes. The Ulysses data have shown that SEPs can easily reach high latitudes. For example, a comparison of intensities of $>70 \mathrm{MeV}$ protons at Ulysses

Correspondence to: S. Dalla (s.dalla@ic.ac.uk) and in the ecliptic shows that energetic particle access to high latitudes is a normal occurrence rather than an exceptional one (McKibben et al., 2001).

The Ulysses observations pose a challenge to our understanding of SEP acceleration and propagation. The most favoured explanation for long duration SEP events (so-called gradual events) is that particles are accelerated at the shock driven by a coronal mass ejection (CME) in the corona and interplanetary space. Within this model, the most important factor in determining the intensity profile of SEP events is the magnetic connection to the shock that accelerates the particles. Interplanetary scattering is thought to be of minor importance in determining the observed profiles (Reames, 1999). Therefore, SEPs would be expected to reach high latitudes in cases where the CME shock extends to the highlatitude magnetic field lines.

In their analysis of the Bastille event, Zhang et al. (2003) showed that the CME shock did not extend to high latitudes in this event. They explained the large delays in particle onsets at Ulysses and the large apparent path length travelled by the particles as due to significant cross-field diffusion. They suggested that the latter might arise as a combination of diffusion by turbulence in the interplanetary magnetic field and the random walk of the heliospheric magnetic field lines.

As an alternative to cross field diffusion, it has been suggested that particles might reach high-latitude magnetic field lines close to the Sun. Models have shown that magnetic field lines connecting high latitudes with low-latitude active regions exist in the solar corona (Neugebauer et al., 2002).

Onset time analysis can provide information on the propagation of the first arriving particles prior to reaching a spacecraft. If acceleration occurs simultaneously at all energies and scattering is low, faster particles will reach a spacecraft earlier than slower ones. A plot of particle onset times in several energy channels versus the inverse particle speed will be characterised by a slope giving the experimental path length. 
Table 1. SEP events at high latitudes

\begin{tabular}{cccccccccc}
\hline event $\mathrm{n}$ & year & date & doy & SXR onset & CME onset & flare loc. & SXR class & $\mathrm{R}_{\text {Ulysses }}$ (AU) & Ulysses heliolat \\
\hline 1 & 2000 & $14 \mathrm{Jul}$ & 196 & $10: 03$ & $10: 54$ & N22 W07 & X 5.7 & 3.17 & -62.1 \\
2 & 2000 & $12 \mathrm{Sep}$ & 256 & $11: 31$ & $11: 54$ & S17 W09 & M 1.0 & 2.80 & -70.9 \\
3 & 2000 & 8 Nov & 313 & $22: 42$ & $23: 06$ & N10 W77 & M 7.4 & 2.41 & -79.3 \\
4 & 2001 & 15 Aug & 227 & - & $23: 54$ & - & - & 1.63 & +63.1 \\
5 & 2001 & 24 Sep & 267 & $09: 36$ & $10: 30$ & S16 E23 & X 2.6 & 1.90 & +78.2 \\
6 & 2001 & 4 Nov & 308 & $16: 03$ & $16: 35$ & N06 W18 & X 1.8 & 2.18 & +77.6 \\
7 & 2001 & 22 Nov & 326 & $20: 22$ & $20: 30$ & S25 W67 & M 3.8 & 2.31 & +73.7 \\
8 & 2001 & 26 Dec & 360 & $04: 32$ & $05: 30$ & N08 W54 & M 7.1 & 2.54 & +66.7 \\
9 & 2002 & 21 Apr & 111 & $00: 59$ & $01: 27$ & S14 W84 & X 1.5 & 3.28 & +47.9 \\
\hline
\end{tabular}

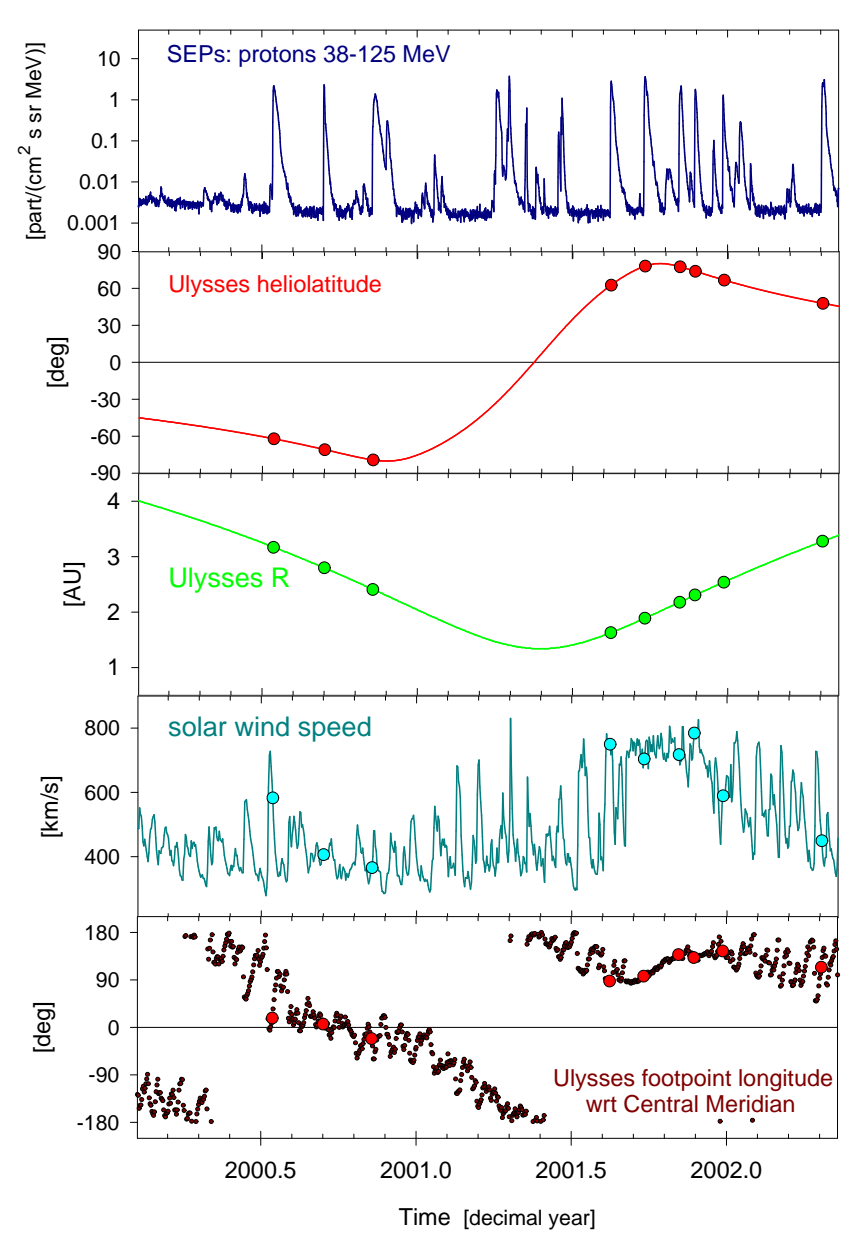

Fig. 1. Overview of Ulysses SEP high-latitude measurements. From top to bottom panels: SEP fluxes for protons $32-125 \mathrm{MeV}$ (COSPIN/KET); Ulysses heliographic latitude; Ulysses distance from the Sun; solar wind speed (SWOOPS); longitude of the nominal footpoint of a Parker spiral field line through Ulysses, with respect to Central Meridian (the footpoint position is calculated by using the measured solar wind speed). The dots on the plot are at the start time of the 9 large SEP events considered in the onset time analysis.
In the absence of scattering this will coincide with the physical distance travelled by the particles between the acceleration region and the spacecraft. The apparent time of release of the particles from the acceleration region can also be derived.

Onset time analysis of Wind data has shown that the first electrons arriving at a near-Earth spacecraft have typically travelled a distance equal to the length of a Parker spiral magnetic field line connecting the spacecraft to the Sun, i.e. approximately 1.2 AU (Krucker et al., 1999). Their propagation appears to be scatter-free. Protons, on the other hand, show two distinct types of behaviour: in some cases they travel $\sim 1.2$ AU but are released approximately $1 \mathrm{~h}$ after electrons; in other cases they travel a length of $\sim 2 \mathrm{AU}$ but appear to be released simultaneously with electrons (Krucker and Lin, 2000).

In this paper we present an onset time analysis of highlatitude SEP events. We use data from the Ulysses COSPIN experiment and compare the onsets at high latitudes with those observed in the ecliptic by Wind 3DP and SOHO COSTEP. We discuss whether the results of the onset time analysis can help to identify the mechanism by which SEPs reach high latitudes. Experimental values of the path length for high latitude SEP events could be used to constrain models of solar particle and cosmic ray propagation.

\section{Data analysis}

Figure 1 shows an overview plot of the Ulysses SEP measurements between February 2000 and May 2002. During this time, Ulysses travelled over the South Pole of the Sun, then moved northwards, crossing the ecliptic and passing over the North Pole (see second panel in Fig. 1). The distance of the spacecraft from the Sun in this period varied between 1.3 and $4.0 \mathrm{AU}$.

The top panel in Fig. 1 shows several large SEP events being detected at high heliographic latitudes. We selected for our study SEP events producing large count rates in the KET 38-125 MeV proton channel and obtained a list of 9 events. The start times of the SEP events are indicated by dots in 

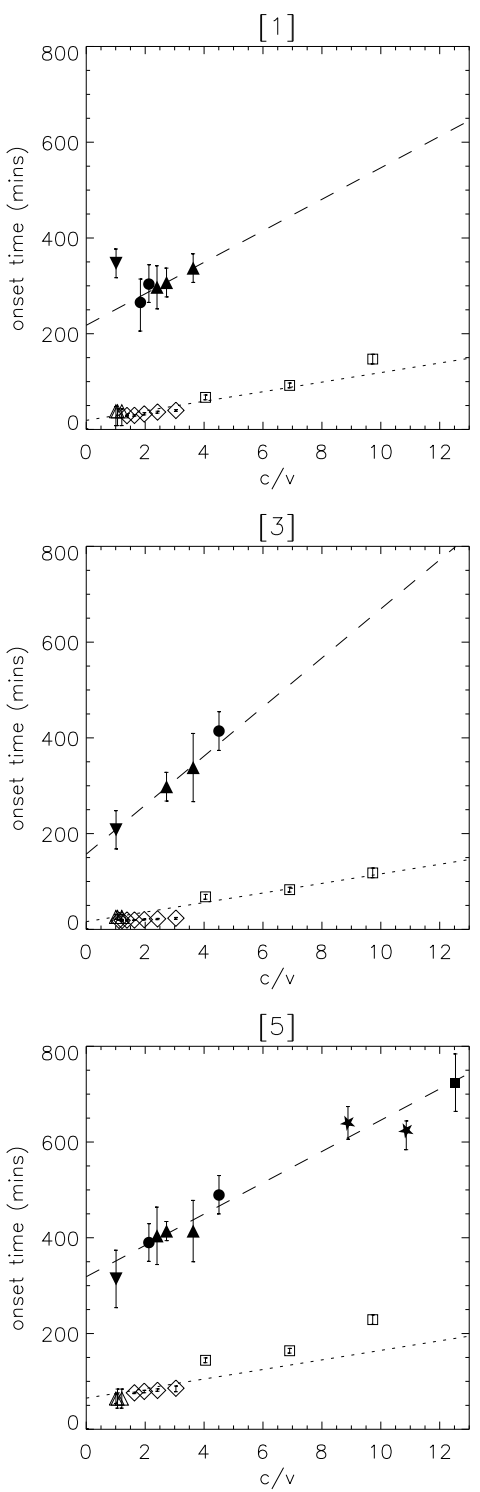
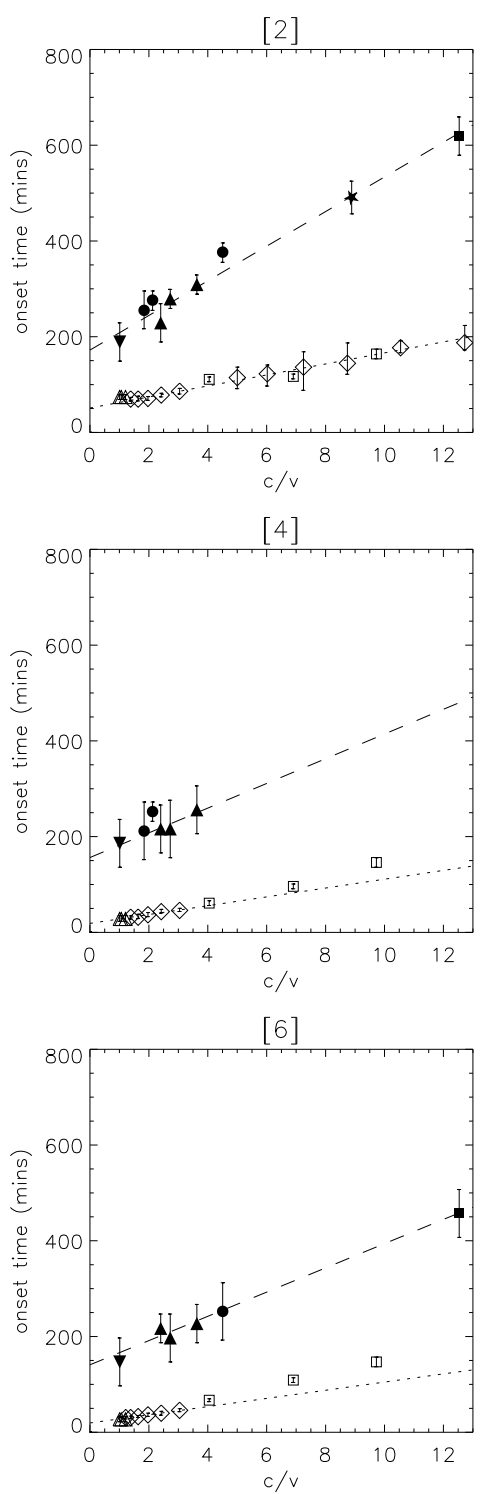

Fig. 2. Plots of onset time versus $c / v$ near Earth (empty symbols) and at Ulysses (filled symbols). Details of the 9 events are given in Table 1 . Onset times are given in minutes from the start time of the GOES SXR flare.

the panels of Fig. 1. Details of the events, and of their associated Soft-X-Ray flares and CMEs, are given in Table 1. During the events, Ulysses' distance from the Sun was between 1.63 and 3.28 AU, and for all but one event its latitude was in magnitude between $60^{\circ}$ and $80^{\circ}$. Four of the events took place while the spacecraft was immersed in fast solar wind (over the North pole of the Sun), three in slow solar wind and two in intermediate speed wind (around $600 \mathrm{~km} / \mathrm{s}$ ).

\subsection{Onset time analysis}

A particle of energy $E$ released from the acceleration region at the time $t_{\text {Sun }}$ and travelling scatter-free a distance $L$ will reach the detecting spacecraft at the time:

$t_{\mathrm{s} / \mathrm{c}}(E)=t_{\mathrm{Sun}}+L / v(E)$, where $v$ is the particle speed. If particles in all the energy ranges considered are released simultaneously and travel the same distance, i.e. the quantities $t_{\text {Sun }}$ and $L$ are independent of energy, onset times plotted versus $1 / v$ lie on a straight line. The slope of the line gives the experimental path length and the intercept with the $y$-axis gives the time of particle release.

\subsection{Instruments and onset time measurement}

We measured onset times from energetic particle data recorded out of the ecliptic by the Ulysses COSPIN experiment (Simpson et al., 1992) and near the ecliptic by Wind/3DP (Lin et al., 1995) and SOHO/COSTEP (MüllerMellin et al., 1995). The COSPIN experiment consists of several separate energetic particle detectors. We measured electron onset times from the HET instrument (electrons 1-3 

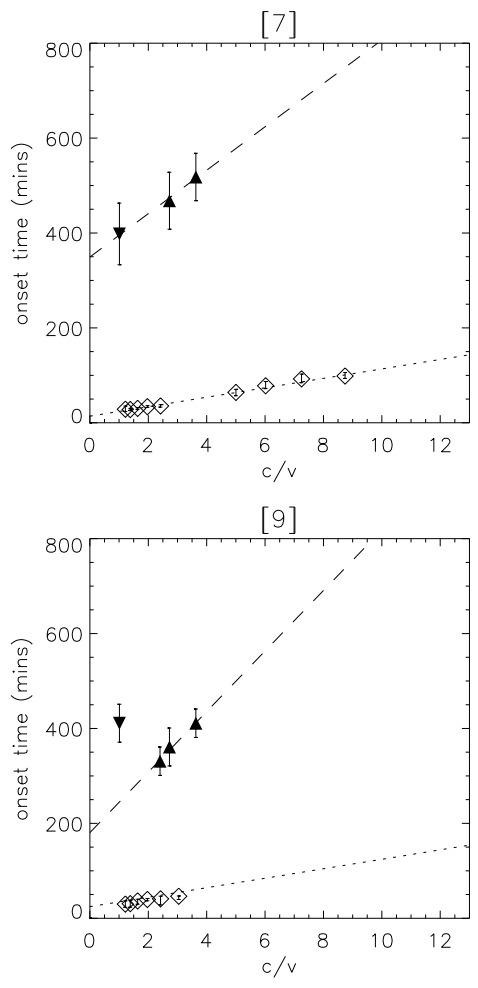

Fig. 2. continued...

$\mathrm{MeV}$ ). For protons, we used channels from the HET, LET, ATs and HFT. Most of the HET proton channels are contaminated by electrons during the onset of SEP events. For some of these channels we were able to subtract the electron contributions. For Ulysses, we used spin-averaged data. The pitch angle of the particles responsible for the measured onset might not be zero. If we use Eq.(1), a nonzero pitch angle would result in the experimental path length being larger than the actual path length by approximately a factor of $1 / \cos \theta$, with $\theta$ representing the pitch angle of the first arriving particles. A further complication to the above consideration is the decrease in particle pitch angle as particles travel away from the Sun in the interplanetary magnetic field (focussing). Wind/3DP electron data at zero pitch angle and SOHO/COSTEP electron and proton data were used.

We determined onset times by following the procedure outlined by Krucker et al. (1999). This involves subtraction of the pre-event background and calculation of its standard deviation $\sigma$. The upper limit $t_{\text {upper }}$ to the onset time is the time at which the particle count rate reaches $4 \sigma$. The onset time $t_{\text {onset }}$ is the first time prior to $t_{\text {upper }}$ for which particle counts are above zero. The error bar on each value of the onset time is given by 2 ( $\left.t_{\text {upper- }}-t_{\text {onset }}\right)$.

Onset times were determined from 10- or 20-min averaged data for Ulysses and from 64-s averages for Wind data. For a channel measuring particles of energy in the range between $E_{\min }$ and $E_{\max }$, the measured onset time has been assigned to the speed $v\left(E_{\max }\right)$, assuming that the fastest particles arrive first. Some of the Ulysses channels have a wide energy

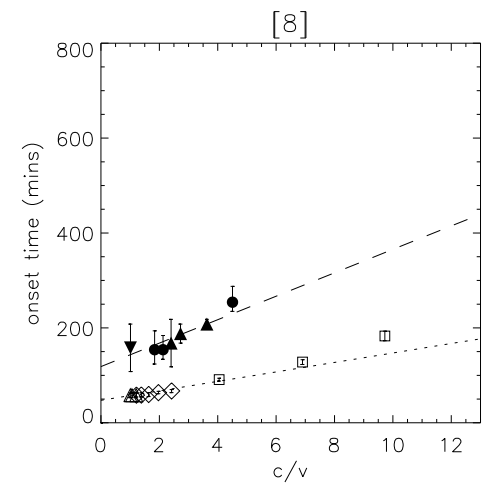

$$
\begin{aligned}
& \diamond \text { Wind/3DP-electrons } \\
& \triangle \text { SOHO/COSTEP-electrons } \\
& \square \text { SoHO/COSTEP-protons } \\
& \text { V Ulysses/HET-electrons } \\
& \text { \Ulysses/HET-protons } \\
& \text { - Ulysses/KET-protons } \\
& \text { - Ulysses/LET-protons } \\
& \text { * Ulysses/HFT/AT-protons }
\end{aligned}
$$

range, which would result in a large error in the determination of $c / v$. These channels have only been used when it was possible to verify from other higher energy channels that particles of energy $E_{\max }$ were present.

\section{Results}

Figure 2 shows plots of onset times versus $c / v$ for the Ulysses high-latitude events, compared with in-ecliptic measurements (filled symbols $=$ Ulysses; empty symbols $=$ Wind and SOHO). In the plots, $t=0$ is the time of onset of the SXR flare associated to the events.

For Ulysses, data for the low values of $c / v$ are from the HET and KET instruments, and for large values of $c / v$ from the LET, HFT and ATs. For several events it was not possible to determine the onsets properly in the low energy channels, because pre-event fluxes were not at background level. Measuring the onset time in these cases would result in artificially large onset times.

A linear fit to the Ulysses data points is shown in the plots of Fig. 2. This fit gives the largest weight to the data points from the HET and LET particle channels because they have the best statistics. For some events, the data point corresponding to HET electrons (filled triangle down) was not included in the fit, because it does not lie on the same line as the proton data points.

The fact that the data point corresponding to electrons does not line up with the proton onsets in event 1 (Bastille event) was interpreted by Zhang et al. (2003) as a result of the trans- 
port of particles being rigidity dependent. The plots of Fig. 2 show that in most cases the electron onset time lies on a common line with proton onsets. This, however, does not happen in events 1 and 9, which have the largest distances of Ulysses from the Sun.

Wind/3DP electron onsets are consistent with scatter-free propagation along a field line of length $\sim 1.2$ AU. For event 3 , this is not the case: in very large events with very impulsive onsets, the velocity dispersion can be hidden by instrumental counts at low energies produced by high energy electrons that scatter out of the detector before they lost all their energy.

It should also be noted that for event 7 , a second flare followed the flare whose details are given in Table 1. This was an M 9.9 flare at 22:32 UT located at S 15W 34. For Ulysses onsets, it cannot be determined whether the particles detected were associated to the first or second flare. Taking the second solar event as the source of particles would reduce the value of $t_{\text {Sun }}$ by $130 \mathrm{~min}$, and the difference in latitude between flare and Ulysses would decrease by $10^{\circ}$. The speed of the CME associated to the second flare is the same as that of the CME associated to the first one $(1400 \mathrm{~km} / \mathrm{s})$. If the second flare were the source of the particles at Ulysses, this would not significantly change the conclusions of the analysis below.

We see from Fig. 2 that onsets at high latitudes are characterised by release times much later than in the ecliptic, and by larger path lengths. The values of $t_{\text {Sun }}$ and $L$ obtained from the fitting of the plots in Fig. 2 will be discussed in Sect. 3.2. First, in Sect. 3.1 below, we present a test of our method for determining onset times.

\subsection{Test of onset time determination method}

The onset time determined from a particle channel can be influenced by several factors which include: the background in the channel, the geometric factor of the instrument and the viewing direction. When one looks at the main feature of the plots in Fig. 2, the very large delay in onset at Ulysses compared to in-ecliptic onset, one may ask whether there is any possibility that this delay might be an instrumental effect rather than being caused by a physical process.

We observe that in the plots of Fig. 2 there is generally good agreement between the onset times obtained at Ulysses from separate instruments, for example, from the HET (filled triangles) and KET (filled circles) instruments.

As a test for our method we analysed onsets for an SEP event taking place when Ulysses was at low latitudes and small radial distances from the Sun. For such a location we would expect the Ulysses data to show delays similar to those observed by Wind.

We selected for the test the event of 15 June 2001, during the Ulysses fast latitude scan (see Fig. 1). On this day Ulysses was located at $1.36 \mathrm{AU}$ from the Sun, at a latitude of $22.3^{\circ} \mathrm{N}$ and $77^{\circ}$ west of the Sun-Earth line. There is no flare association for the event, but a halo CME with onset at 15:56 was observed by $\mathrm{SOHO} / \mathrm{LASCO}$. This suggests that a flare might have taken place on the far side of the Sun.

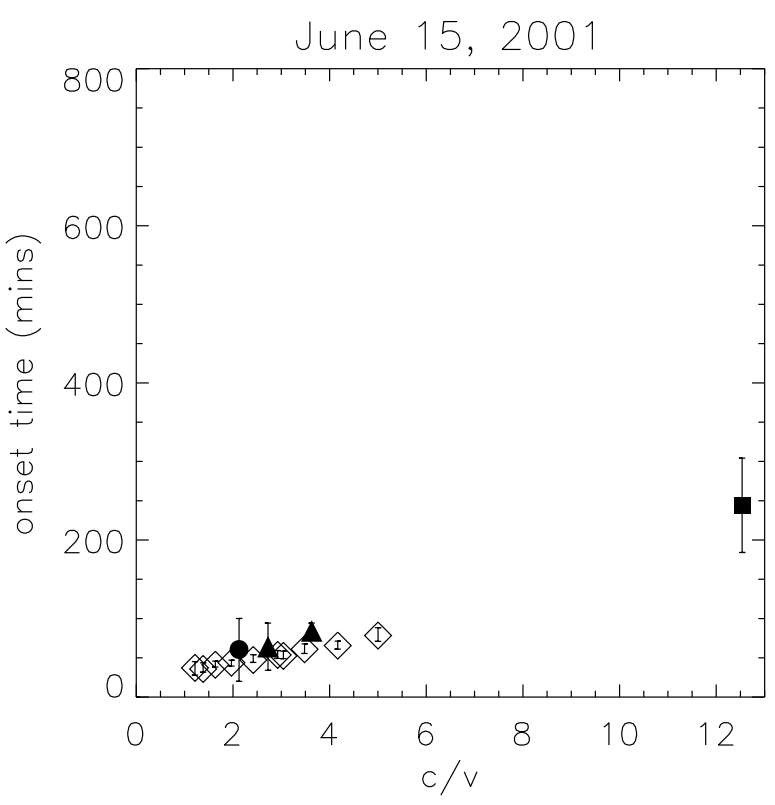

Fig. 3. Onsets at Ulysses and Wind for the 15 June 2001 event, when Ulysses was at low latitudes.

Figure 3 shows onsets at Ulysses and Wind for the low latitude event of 15 June. The Ulysses data points are in good agreement with the Wind data points. This confirms that the late release times of the plots in Fig. 2 are not an instrumental effect but are due to a physical process.

3.2 Correlations of path length and release time with event parameters

In this section we plot the path length $L$ and release time $t_{\text {Sun }}$ from our fitting, versus several parameters characterising the events. The values obtained from the fitting are first plotted versus event number in Fig. 4. Ulysses measurements of the path length have very large error bars in cases where no data points were available at low energies.

For each event we have calculated the nominal length $L_{p}$ of the Parker spiral magnetic field line that would connect Ulysses to the Sun, using the measured solar wind speed. A plot of the experimental path length $L$ versus $L_{p}$ is given in Fig. 5a, where the dotted line corresponds to $L=L_{p}$. The ratio of experimental path length to Parker length varies between 1.06 and 2.45 for our data points. In Fig. $5 b$ the ratio $L / L_{p}$ is plotted versus the release time. There is no clear correlation between these two parameters.

We then looked for correlations between our fitting coefficients and measures of the separation between the footpoint at the Sun of the observing spacecraft and the location of the flare. We calculated a nominal location of the spacecraft magnetic footpoint by assuming a Parker spiral magnetic field line and using the measured solar wind speed. Figure 6 shows a plot of the release time versus: (a) the difference in longitude $|\Delta \phi|=\left|\phi_{\text {footpt }}-\phi_{\text {flare }}\right|$; (b) the great cir- 

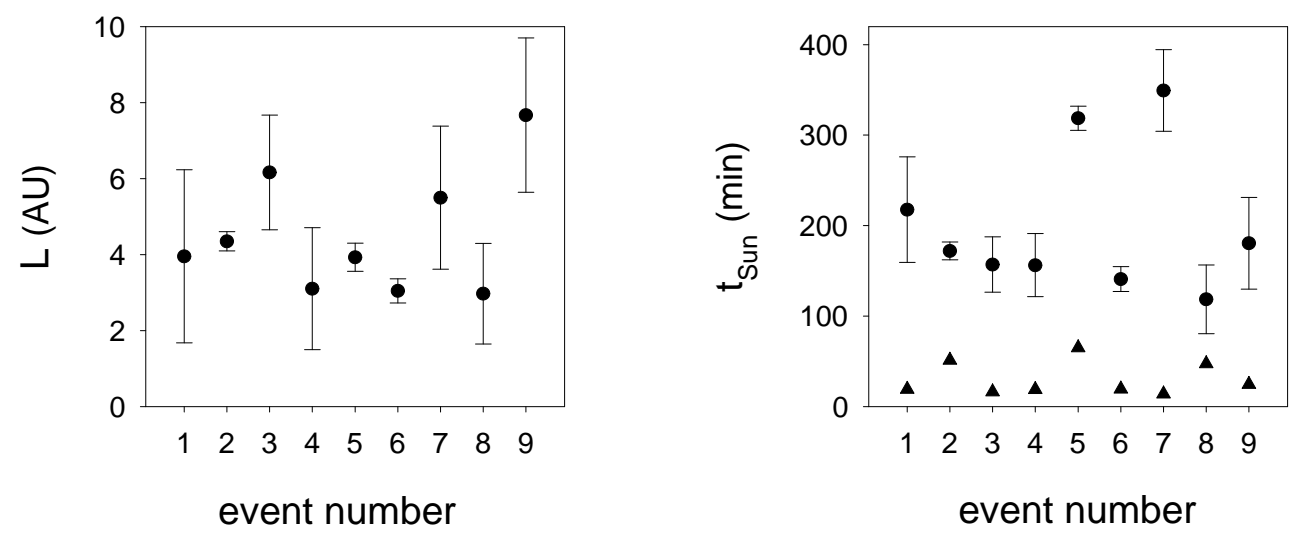

Fig. 4. Values of path length $L$ and release time $t_{\text {Sun }}$ obtained from fitting of the onset times curves, versus event number. Ulysses data points are circles and Wind are triangles. The error bars for Wind data points are smaller than the size of the triangles. Release times are in minutes from the onset of the SXR flare.
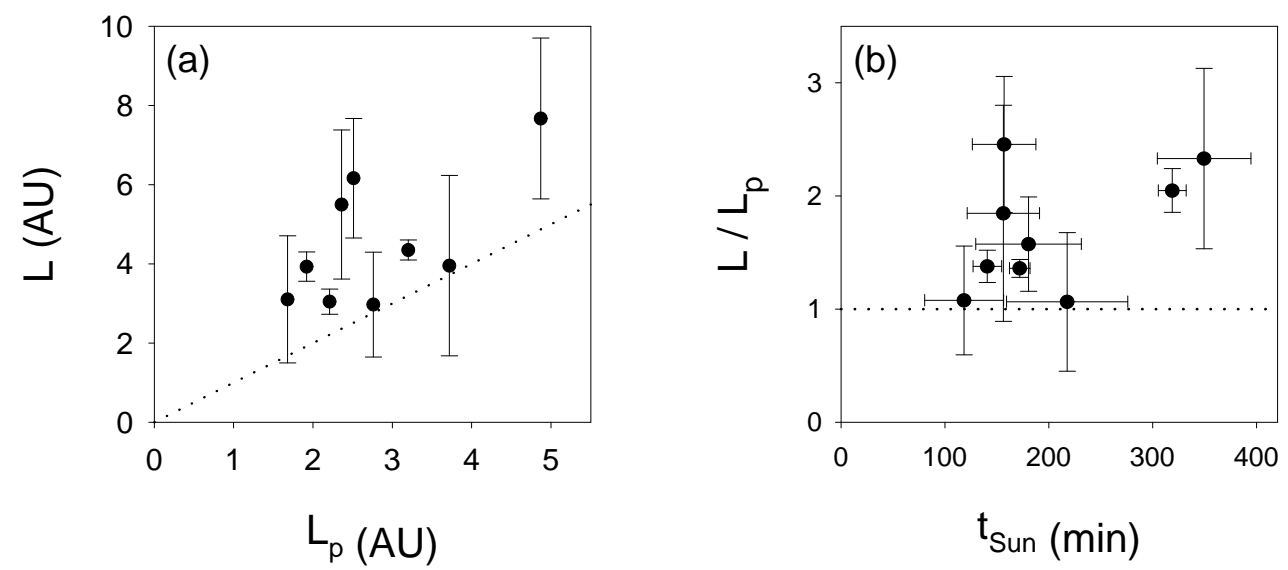

Fig. 5. Plot of (a) experimental path length versus the length $L_{p}$ of a Parker spiral connecting Ulysses to the Sun; and (b) the ratio $L / L_{p}$ versus $t_{\text {Sun. }}$. The dotted lines correspond to $L=L_{p}$.

cle angular separation $|\Delta \alpha|$ between spacecraft footpoint and flare; and (c) the difference in latitude $|\Delta \theta|=\left|\theta_{\text {footpt }}-\theta_{\text {flare }}\right|$. Panel (a) shows that there is no correlation between $t_{\text {Sun }}$ and the difference in longitude between spacecraft footpoint and flare in the ecliptic (triangles), as already reported (Krucker and Lin, 2000).

Surprisingly, panels (c) and (b) of Fig. 6 show a better correlation between $t_{\text {Sun }}$ and the latitudinal difference $|\Delta \theta|$ than between $t_{\text {Sun }}$ and the angle between flare and spacecraft footpoint $|\Delta \alpha|$. We then plotted the ratio $L / L_{p}$ versus $|\Delta \theta|$ and obtained panel (d) of Fig. 6. Here, one can observe that the data points close to $L / L_{p}=2.2$ are around $|\Delta \theta|=95$ and $4 / 5$ of those close to $L / L_{p}=1.3$ are near to $|\Delta \theta|=60$.

We now look for correlations between the delay in release and the speed of the CME associated to the events, as well as the speed of the CME driven shock. All 9 SEP events were associated with fast CMEs. We found in the SOHO LASCO CME catalogue (available at http://cdaw.gsfc.nasa. gov/) CME onset times (times of first appearance in C2) and CME speeds derived from linear fits of coronograph ob- servations. Of the 9 events considered, 7 were associated with halo CMEs and the position angle of the CME central axis could not be determined. A shock was detected near Earth in all 9 events (ACE shock list, http://www.bartol.udel. edu $\sim$ chuck/ace/ACElists/obs_list.html). At Ulysses, a shock was detected in 3 of the 9 events (R.J. Forsyth, private communication, 2002). From the CME onset time and the arrival time of shocks at ACE/Ulysses we derived an average transit speed of shocks. The events for which a shock reached high latitudes have the largest transit speeds in the ecliptic. For the latter events the average transit speed at high latitudes is $970 \mathrm{~km} / \mathrm{s}$ and in the ecliptic plane $1300 \mathrm{~km} / \mathrm{s}$.

Figure 7 shows an analysis of correlations between the measured $t_{\text {Sun }}$ and: (a) the CME speed from coronograph observations; (b) the average shock speed from in-ecliptic data; (c) the average shock speed from high-latitude data. There is no indication from these plots that shorter release times might be associated with the faster CMEs. In other words, we do not find the correlation $t_{\text {Sun }} \propto 1 / v_{\text {shock }}$ which would be expected if the delay in release were due to the time taken 

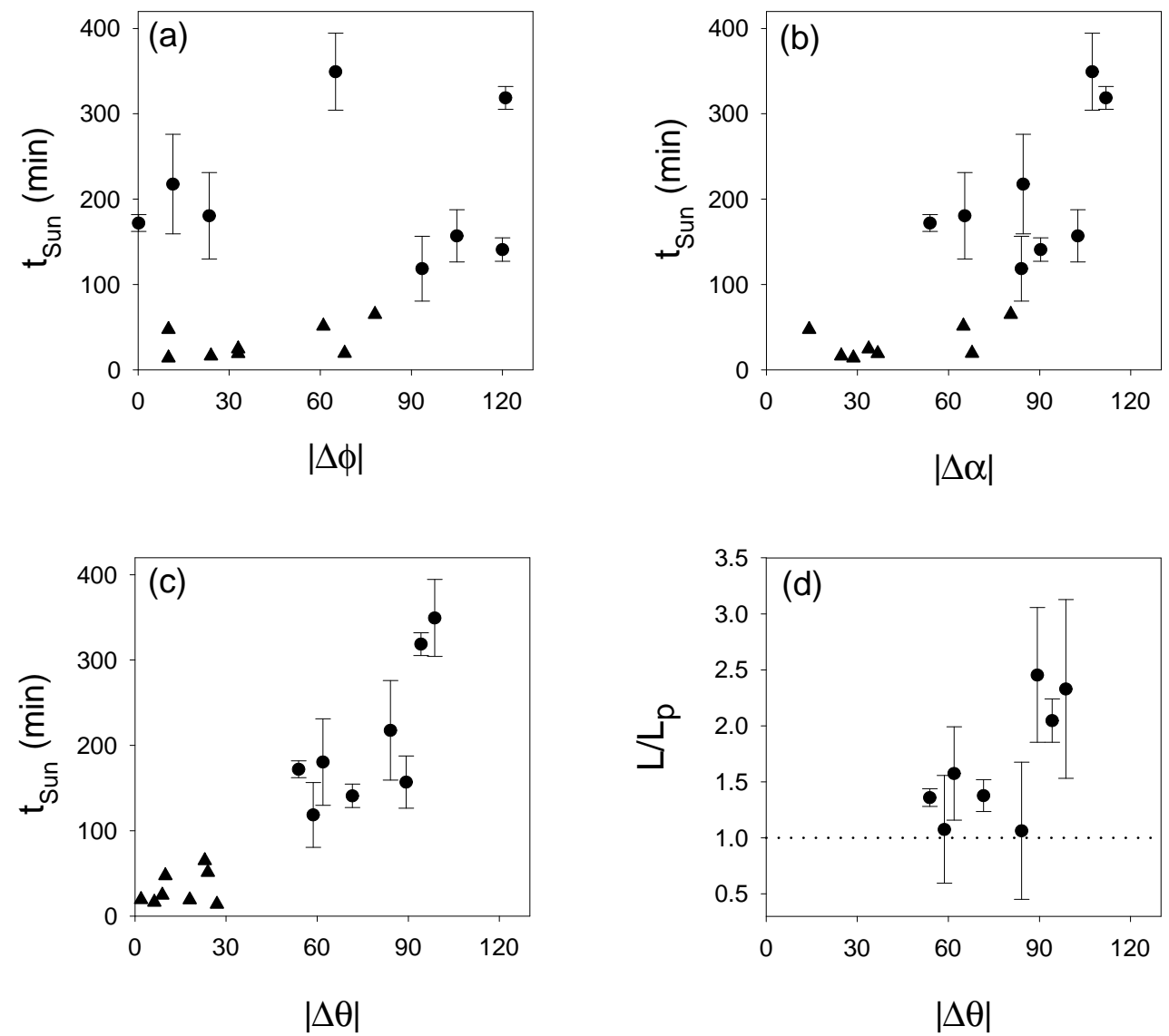

Fig. 6. Release time $t_{\text {Sun }}$ versus angular separations between the spacecraft footpoint and the flare locations. Circles are Ulysses data points and triangles are Wind data points. (a) $t_{\text {Sun }}$ versus difference in longitudes $|\Delta \phi|=\left|\phi_{\text {footpt }}-\phi_{\text {flare }}\right|$; (b) versus great circle angular separation $|\Delta \alpha|$ between footpoint and flare; (c) versus difference in latitude $|\Delta \theta|=\left|\theta_{\text {footpt }}-\theta_{\text {flare }}\right|$. Also given in panel (d) is the ratio $L / L_{p}$ versus $|\Delta \theta|$. Plots are given for only 8 SEP events because the flare location was not known for 1 event.
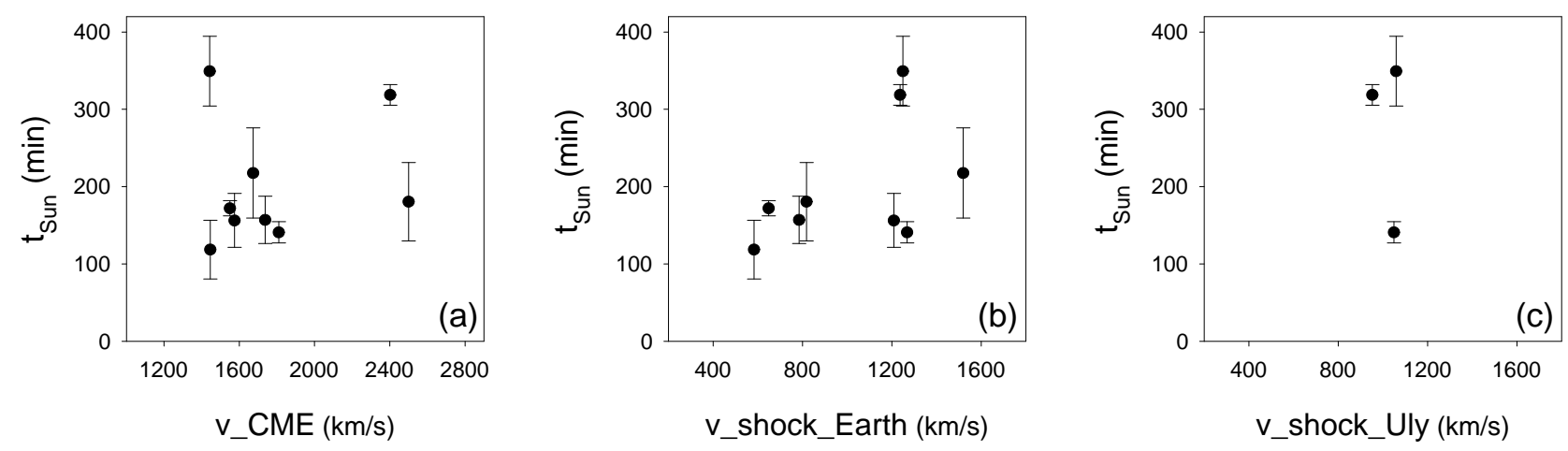

Fig. 7. Release time from Ulysses measurements versus (a) the speed of the CME from SOHO/LASCO coronograph observations; (b) the average transit speed of the CME driven shock in the ecliptic; (c) the average transit speed of the CME driven shock at high latitudes.

by the CME driven shock to reach the high-latitude magnetic field lines. We have also plotted $t_{\text {Sun }}$ versus the ratio of the angular separation $|\Delta \alpha|$ to the shock speed: we find no correlation when we use the coronograph CME speed and the average in-ecliptic shock transit speed.

In Fig. 8 we plot $t_{\text {Sun }}$ and $L$ versus the solar wind speed at Ulysses. It is known that magnetic field fluctuations have larger amplitudes in the high speed solar wind (Forsyth et al., 1996). If the reason for the measured path lengths being larger than the Parker spiral length were due to magnetic field turbulence, a dependence of $L / L_{p}$ with the solar wind speed would be expected. As can be seen from panel (a) of Fig. 8, 

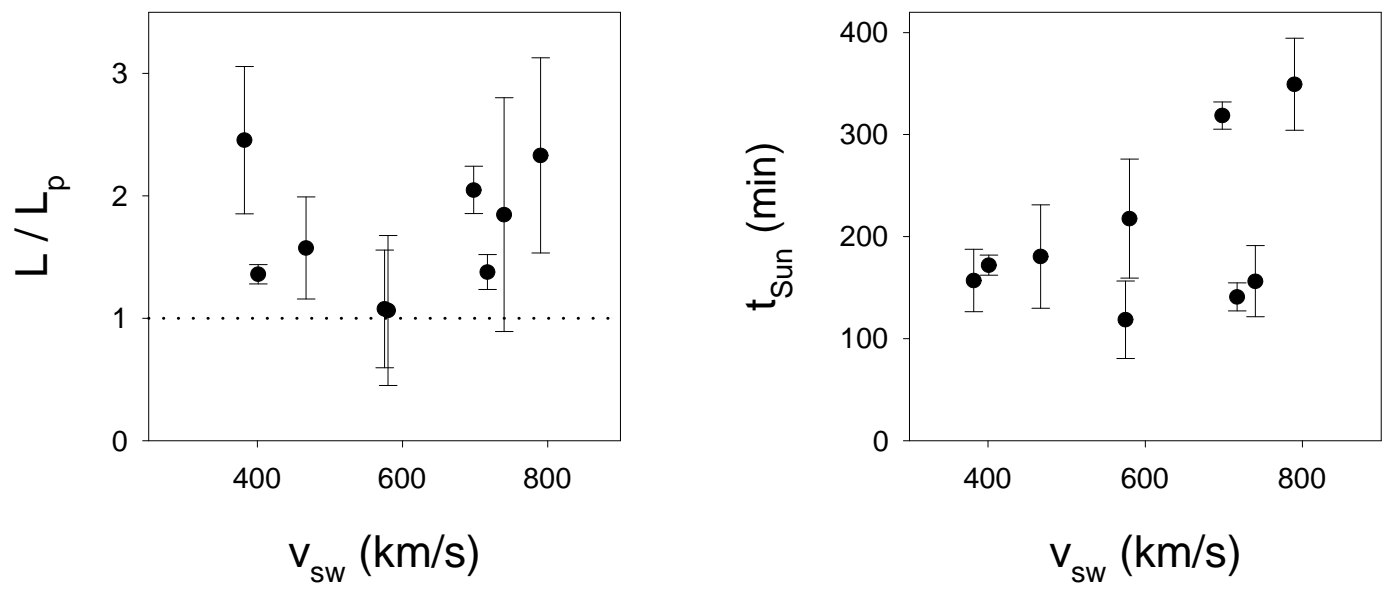

Fig. 8. Path length $L$ and release time $t_{\text {Sun }}$ versus solar wind speed at Ulysses.

this is not observed. The release time shows no correlation with the solar wind speed.

\section{Discussion}

Our analysis has shown that the release of particles to high latitudes is consistently delayed with respect to in-ecliptic release. It would seem natural to interpret this delay within the CME shock acceleration paradigm, as the time needed for the shock to reach the high latitude-magnetic field lines. However, we do not find the expected correlation between delay and several measures of the inverse of the shock speed. These include the inverse of the CME speed derived from coronograph observations, of the average transit speed of the CME shock between the Sun and Earth, and of the average transit speed for arrival at Ulysses for those shocks that reach high latitudes ( 3 out of 9).

Recently, Simnett et al. (2002) interpreted the delay in release observed in ACE electron data as due to the time a CME shock takes to reach altitudes in the solar corona of a few solar radii. The analysis of release delays at high latitudes is complicated by the fact that details of the 3-dimensional propagation of shocks in the solar corona are not known. In other words, we do not know how a CME driven shock would propagate in both latitude and height. Consider a shock front moving at $1000 \mathrm{~km} / \mathrm{s}$, the measured average travel speed to Ulysses. Such a shock would take $18 \mathrm{~min}$ to travel $90 \mathrm{de}-$ grees in latitude along the solar surface, or 23 min to travel to a height of 3 solar radii at a fixed latitude. The delays observed at Ulysses of 120-350 min from the flare onset appear too large to be associated to a travel time of CME shocks to high latitudes, and would correspond to a typical shock being at heights greater than 10 solar radii.

Our analysis shows that the delay in particle release appears to correlate with the difference $|\Delta \theta|$ between the latitude of the spacecraft and the latitude of the flare (Fig. 6c). This parameter organises the release times better than the total angular separation between the spacecraft footpoint and flare. The ratio between measured path length and length of the Parker spiral is close to 1.3 for $|\Delta \theta|$ around $60^{\circ}$, and close to 2.2 for $|\Delta \theta|$ around $95^{\circ}$. The latter findings seem to point towards cross field diffusion being the fundamental mechanism by which particles reach high latitudes. This has been suggested by Zhang et al. (2003), who showed that, in the Bastille event, the proton anisotropy was directed at an angle to the magnetic field direction. Analysis of galactic cosmic ray data at Ulysses and near Earth at solar maximum also reached a similar conclusion (McKibben et al., 2003). Recent work on comparing radio emission at the local plasma frequency at a spacecraft and energetic particle data, suggested that particles must undergo lateral transport (Cane and Erickson, 2003).

The possibility that the observed delay at Ulysses might be due to the time taken by a CME shock to reach field lines connecting to the spacecraft cannot, however, be completely ruled out. It is possible that the lack of correlation of $t_{\text {Sun }}$ with CME shock speed might be due to the shock propagation conditions in the corona varying in the different events. The variable latitudinal extent of CMEs in the events might also play a role.

As far as the cross field diffusion hypothesis is concerned, modelling of particle propagation would be required to assess which scattering parameters can give the observed delays. Up to now, this kind of study has been carried out within the context of the focussed transport equation, which only describes propagation along the magnetic field (Kallenrode and Wibberenz, 1990). The latter study also showed that the finite duration of the particle injection can influence particle onsets.

We have also found no correlation of our fitting parameters with the solar wind speed measured at Ulysses. One possible explanation for this finding could be as follows. If we think of the SEPs as being released at low to mid latitudes and slowly diffusing in latitude as they move away from the Sun, we would expect them to be moving in slow solar wind for most of the time. The fact that Ulysses is immersed in fast 
solar wind would, therefore, not play an important role.

Fisk (1996) suggested that heliospheric magnetic field lines might be undergoing a systematic latitude excursion due to the motion of their footpoints on the solar wind source surface caused by the differential rotation of the Sun. The latitude excursion of the field lines can be several tens of degrees. Within this model, solar energetic particles would reach a spacecraft at high latitudes through a direct connection to the particle source at low solar latitudes by the Fisk heliospheric magnetic field. Since the length of the heliospheric magnetic field lines is approximately the same for both Parker and Fisk models, we would expect that the time of particle release be independent of the latitude of observers. The latitude dependence of $t_{\text {Sun }}$ seems to rule out the possibility of direct connection by the Fisk magnetic field model.

Acknowledgements. We acknowledge use of the Ulysses Data System. S.D. acknowledges support from PPARC through a PostDoctoral Fellowship. M.Y.H. thanks ESA for the current research Fellowship.

Topical Editor R. Forsyth thanks three referees for their help in evaluating this paper.

\section{References}

Cane, H. V. and Erickson, W. C.: Energetic particle propagation in the inner heliosphere as deduced from low frequency $(<100$ $\mathrm{kHz}$ ) observations of type III radio bursts, J. Geophys. Res., in press, 2003.

Fisk, L. A.: Motion of the footpoints of heliospheric magnetic field lines at the Sun: Implications for recurrent energetic particle events at high heliographic latitudes, J. Geophys. Res., 101, 15, 547, 1996.

Forsyth, R. J., Balogh, A., Horbury, T. S., et al.: The heliospheric magnetic field at solar minimum: Ulysses observations from pole to pole, Astron. Astrophys. 316, 287, 1996.

Kallenrode, M.-B. and Wibberenz, G.: Influence of interplanetary propagation on particle onsets, Proc. 21st Internat. Cosmic Ray Conf. Adelaide, 5, p. 229, 1990.

Krucker, S., Larson, D. E., Lin, R. P., et al.: On the origin of impulsive electron events observed at 1 AU, Ap. J. 519, 864, 1999.

Krucker, S. and Lin, R. P.: Two classes of solar proton events derived from onset time analysis, Ap. J. 542, L61, 2000.

Lin, R. P., Anderson, K. A., Ashford, S., et al.: A 3-dimensional plasma and energetic particle investigation for the Wind spacecraft, Space Sci. Rev. 71, 125, 1995.

McKibben, R. B., Connell, J. J., Lopate, C., et al.: Ulysses COSPIN observations of the energy and charge dependence of the propagation of solar energetic particles to the Sun's south polar regions, Proc. 27th ICRC, Hamburg, 3281, 2001.

McKibben, R. B., Connell, J. J., Lopate, C. et al.: Ulysses COSPIN observations of cosmic rays and SEPs from the South Pole to the North Pole of the Sun during solar maximum, Annales Geophys., this issue, 2003.

Müller-Mellin, R., Kunow, H., Fleissner, V., et al.: COSTEP: Comprehensive suprathermal and energetic particle analyser, Sol. Phys. 162, 483, 1995.

Neugebauer, M., Liewer, P. C., Smith, E. J., et al.: Sources of the solar wind at solar activity maximum, J. Geophys. Res. 107, 1488, doi: 10.1029/2001JA000306, 2002.

Reames, D. V.: Particle acceleration at the Sun and in the heliosphere, Space Sci. Revs. 90, 413, 1999.

Simpson, J. A., Anglin, J. D., Balogh, A., et al.: The Ulysses cosmic ray and solar particle investigation, Astron. Astrophys. Suppl. Ser. 92, 365, 1992.

Simnett, G. M., Roelof, E. C., and Haggerty, D. K.: The acceleration and release of near-relativistic electrons by Coronal Mass Ejections, Astrophys. J. 579, 854, 2002.

Zhang, M., McKibben, R. B., Lopate, C., et al.: Ulysses observations of solar energetic particles from the July 14, 2000 event at high heliographic latitudes, J. Geophys. Res., 108, 1154, doi: 10.1029/2002JA009531, 2003. 